\title{
Changes in the R\&D process and consequences on drug pricing
}

\author{
Rubin Zareski \\ Faculty of Pharmacy, Ss. Cyril and Methodius University, Mother Theresa 47, 1000 Skopje, North Macedonia
}

\section{Introduction}

A new paradigm of the so called "Circular economy and resource efficiency" economy is evolving. Optimisation, sustainability, long term efficiency, customization are becoming widely spread pillars of a new development Models. Pharmaceutical business is not spared from these systematic changes. Moreover, the pharmaceutical industry, regulator, funds, payers and patients are under growing pressure to adopt very fast to these emerging needs. Old pricing methodologies rely to a significant extent to costs and consequently on price reduction affecting all parts of supply and delivery chain. In the eyes of patients and other stakeholders, the pharmaceutical industry exists to discover new medicines that go on to become standard treatments. The faltering economics of $R \& D$ productivity are jeopardizing that mission: $R \& D$ expenditure is not delivering. Although number of facts are saying that now is the right time (maybe also last chance) to institutionalize the process of the "holly trinity", R\&D, HTA, reference pricing methodology, major dilemma is whether the approach should be driven by market forces or by regulations, or by mix.

\section{Redefined or revolutionized approach}

Healthcare consumes $12 \%$ of global GDP, and is a $\$ 6.2$ trillion industry. Spend is growing at average of $3.5 \%$ per annum, which will double expenditure in less than 20 years. By 2020, chronic diseases will account for almost three-quarters of all deaths worldwide. Moreover, it is estimated that by 2030 more than $40 \%$ of the world population will suffer from one or more chronic disorders. By 2080, estimated $50 \%$ of world GDP will be spend on health costs (OECD analysis). The health care system is under growing pressure as a result. Unless we intervene at short notice in a number of different domains, financial and staffing shortfalls will be inevitable. To ensure that health care system is future proof, we need a new perspective that corresponds more closely to people personal experience.

This route has three game changers. First is to acknowledge the health as a basic condition for participating, playing active role in society a different and broader view than the prevailing biomedical model, which focuses on sickness. Introduction of the concept of "positive health", in which actions are guided by the desires, values and preferences of the individual needs, is to become a focal point. Second, actions of all involved participants must be focused on the notion of QUALY that should replace the limited approach of what someone with an illness is still capable of. Third, to implement the concept of positive health, we must be more knowledgeable and aware of variations between individuals: variations in norms, values and goals, but also in lifestyle, behaviour, environment, genetic disposition, and above all in the body's response to healthy and pathogenic stimuli.

There are two possible alternatives in addressing this situation. First are the so called "Pragmatic

\footnotetext{
* rubin@trust.com.mk
} 
trials" which provide fast and efficient information for decision making by providing evidence for the adoption of treatments into practice (Ford \& Norrie, 2016). Carefully constructed and executed pragmatic trial provides the bridge between research performed for the development of treatments to the evaluation of the effectiveness of treatments in practice. Second solution is to outsource the $R \& D$ to the more efficient, focused, equipped companies and to introduce pay-for-performance Model.

In this new environment, pharmaceutical companies will have to make major shift in the policy implementation by trading price for access to markets. On the other side Governments on the long run in finding a right balance will need to introduce a pharmacoeconomic studies. To make this new approach possible beside the large-scale comparative valuable studies that form the basis of medicine today new research methods and outcome measures will focus on valid and sensitive measures for many disorders that allow us to track and predict outcomes to supplement existing methodologies. What is vital for future $\mathrm{R} \& \mathrm{D}$ is a broad, interdisciplinary approach in which the public/patients, pharmaceutical organisations and co-funding bodies also play an important role mainly by investing in sickness prevention. We should also be studying and applying the concept of personalised prevention. It is also important to research how prevention can be integrated permanently into the social fabric.

\section{New R\&D model}

Considering the fact that about $15 \%$ to $20 \%$ of clinical spend is wasted on efforts that are not valued by key stakeholders, pharmacoeconomists need to focus on:

1. continuing analysis, including assessment of the critical drivers of behavior for each stakeholder (prescriber, pay or/and patient). One very important and specific prerequisite is to have an advanced data infrastructure consisting of existing and new studies and documentation.

2. detailed comparison of competitor labels and clinical data- to further inform which efficacy and safety endpoints matter most, and maps each of the brands against the stakeholder perception

3. understanding of how competitors are perceived by each stakeholder against the most critical factors, which can help identify unmet needs, and

4. profound understanding of real-world data on treatment decisions and outcomes.
Establishment of a common denominator with a clear 'value proposition' needs to be at the core of pricing and re-imbursement submissions. Incremental costs need to consider potential incremental revenues from priority markets (price, positive formulary place, time-to-market) and the potential downside risk of unfavourable comparisons with alternative treatments. IT will in this future play an essential role by assessment of how personalised medicine and prevention will be organised. In many cases, targeted technology will need to be developed, for example intelligent measuring instruments, labs-on-a-chip, nanomedicine and technologies in such areas as genomics, metabolomics and proteomics. Patients, must therefore be involved at an early stage, facilitating both science and enterprise in developing new technological applications based on costeffectiveness methodologies - known as Health Technology Assessment.

\section{Conclusion}

Original new forms of trans-institutional initiatives and teams composed of an unconventional combination of disciplines between university medical centres, universities of technology, social sciences faculties, applied research institutions, and universities of applied sciences, must be extended and broadened in the R\&D design and data analysis. The new scientific outlook on prevention, treatment and care should impact the design and financing of research programmes. At times, that may require us to change the rules, for example so that insurers can also invest in research. New publishing paradigms involving risks analysis and technology assessment and new methods for measuring research output can also help drive innovation in the pursuit of science.

\section{References}

Berggren, R., Fleming, E., Keane, K., Moss, R., 2018. $\mathrm{R} \& \mathrm{D}$ in the age of agile.

McKenzey\&Company, 2010. Invention reinvented.

OECD, WHO, Growth rate average growth in spending in OECD countries 1995-2015.

PWC, Pharma 2020: Virtual R\&D Which path will you take? PWC paper, 2007.

UK Pharmaceutical Industry Competitiveness Task Force, 2012.

WTO, Annual reports 2018.

Zareski, R., 2012. Basics of Pharmacoeconomics. Akademika,

Maced. Pharm. Bull. 66 (Suppl 1) 63 - 64 (2020) 